\title{
Optimal Graft Thickness for Different Sizes of Tympanic Membrane Perforation in Cartilage Myringoplasty: A Finite Element Analysis
}

\author{
Chia-Fone Lee, MD; Jyh-Horng Chen, PhD; Yuan-Fang Chou, PhD; Lee-Ping Hsu, MD; \\ Peir-Rong Chen, MD; Tien-Chen Liu, $\mathrm{PhD}$
}

Objective: The purpose of this study was to determine, using finite element analysis, the optimal graft thickness for cartilage myringoplasty in patients with different sizes of tympanic membrane (TM) perforations. Study Design: We developed a cartilage plateTM-coupled model using high-resolution computed tomography and finite element analysis. The geometric models of the perforated TM were generated using Patran and ANSYS software. Method: Three different sizes of TM perforations $(15 \%, 55 \%$, and $85 \%$, representing small, medium, and large perforations, respectively) were created in the pars tensa. A cartilage plate was used to repair the eardrum perforation, and the new TM-cartilage coupled complex was loaded into our three-dimensional biomechanical model for analysis. The frequency-amplitude responses for different cartilage thicknesses were compared with those for natural TM. Results: Our results show that, first, in cases with $85 \%$ perforation, the frequencyamplitude responses that were most similar to natural TM at lower frequencies were for graft thicknesses of $0.2 \mathrm{~mm}$ and for $0.1 \mathrm{~mm}$ at higher frequencies.

From the Institute of Biomedical Engineering (C.-F.L., J.-H.C.), National Taiwan University, Taipei, Taiwan; the Department of Otolaryngology (C.-F.L., L.-P.H., P.-R.C.), Buddhist Tzu Chi General Hospital, Hualien, Taiwan; the Department of Medicine (c.-F.L., L.-P.H., P.-R.C.), Buddhist Tzu Chi University, Hualien, Taiwan; the Institute of Electrical Engineering (J.-H.c.), College of Electrical Engineering and Computer Science, National Taiwan University, Taipei, Taiwan; the Institute of Mechanical Engineering (Y.-F.C.), College of Mechanical Engineering, National Taiwan University, Taipei, Taiwan; and the Department of Otolaryngology (T.-C.L.), National Taiwan University Hospital, Taipei, Taiwan.

Editor's Note: This Manuscript was accepted for publication December $18,2006$.

This work was supported by a grant from National Taiwan University Hospital to T.-C.L. (grant number NTUH 94A24 and 95A02) and a grant from Buddhist Tzu Chi General Hospital to C.-F.L. (grant number TCRD 9505 and 9506).

Send correspondence to Dr. Tien-Chen Liu, Department of Otolaryngology, National Taiwan University Hospital, Taipei, Taiwan. E-mail: liuent@ha.mc.ntu.edu.tw; or Dr. Peir-Rong Chen, Department of Otolaryngology, Buddhist Tzu Chi General Hospital, Hualien, Taiwan. E-mail: cprong@m53.hinet.net

DOI: 10.1097/mlg.0b013e318031f0e7
Second, in cases with $55 \%$ posterior perforation of the TM, assessment of the predicted vibration amplitude of different thicknesses of the cartilage plate showed that a cartilage plate of less than $0.2 \mathrm{~mm}$ had a frequency response function similar to that of a natural TM in umbo and stapes footplate displacement. Finally, for a central perforation involving $15 \%$ of the TM, a cartilage plate of less than $1.0 \mathrm{~mm}$ showed a frequency response function similar to that of TM in umbo and stapes-footplate displacement. Conclusions: On the basis of our biomechanical analysis, the optimal thickness of a cartilage graft for myringoplasty appears to be 0.1 to $0.2 \mathrm{~mm}$ for medium and large TM perforations. For small perforations, a cartilage of less than $1.0 \mathrm{~mm}$ is a good compromise between mechanical stability and low acoustic transfer loss. Key Words: Cartilage myringoplasty, middle ear biomechanics, mathematical model.

Laryngoscope, 117:725-730, 2007

\section{INTRODUCTION}

Reconstruction of the tympanic membrane (TM) using fascia or perichondrium aims at achieving the normal anatomy and functions of the TM. Fascia, skin, vein, perichondrium, and dura mater have been used for TM reconstruction. $^{1-4}$ To date, temporalis fascia and perichondrium remain the most commonly used materials for closure of TM perforations, and successful reconstruction is anticipated in about $90 \%$ cases of primary tympanoplasty. ${ }^{5}$ The use of cartilage for middle ear reconstruction is not new. ${ }^{6}$ Autogenous cartilage contributes minimally to an inflammatory tissue reaction and is incorporated in the middle layer of the TM, with contributions to the external layer coming from the skin epithelium and to the inner layer from the mucous membrane of the middle ear. Cartilage also provides a firm scaffolding with resistance to infection during the healing period. The occurrence of retraction pockets postoperatively is hindered, and the possibility of recurrent perforation is reduced. Cartilage has numerous advantages over fascia. For example, the 
thickness of cartilage offers stiffness that is more resistant than fascia to anatomic deformation caused by negative middle ear pressure, thus improving long-term anatomic integrity. Although cartilage has demonstrated good results in TM reconstruction, it has the reputation of sacrificing hearing improvement because the middle ear mechanism is loaded with increased mass and stiffness. The increased mass and stiffness of the reconstructed TM raises its acoustic impedance, which alters acoustic transfer characteristics. The extent of this change depends largely on the material properties of the graft, namely, its density and Young's modulus. Reducing the thickness of the cartilage transplant is one approach to improving its sound transmitting properties. ${ }^{7}$ However, sound transmission through the reconstruction is also affected by the shape and location of the TM perforation and, therefore, the surgical technique used.

Finite element (FE) analysis is a computer-simulation technique used in engineering and biomechanical analysis. The technique uses the Ritz method of numerical analysis and minimization of variational calculus to obtain approximate solutions for vibrational systems. Investigations on reconstruction of the middle ear geometry have been reported in several papers. ${ }^{8,9} \mathrm{FE}$ analysis has distinct advantages compared with other approaches in modeling complex biological systems. With use of FE analysis, the geometry, ultrastructural characteristics, and material properties of a complex biological system can easily be modeled. Using the combined technologies of $\mathrm{FE}$ analysis and three-dimensional reconstruction of the middle ear, we developed an FE model of the human middle ear with TM, ossicular bone, and middle ear ligament. ${ }^{10}$ This model was validated by comparing data from it with published experimental measurements, and it was tested in several otologic applications. ${ }^{10-14}$

The purpose of this study was to determine the optimal graft thickness in cartilage myringoplasty for different sizes of TM perforation using FE analysis. To do this, we developed a cartilage plate-TM-coupled model using FE analysis based on our previous work. ${ }^{11}$ The Raleigh damping parameter, $\beta$, of the cartilage plate at different frequencies and the optimal thickness of cartilage were calculated by using FE analysis. ${ }^{11}$ Three different sizes of TM perforation (15\%, 55\%, and $85 \%$ representing small, medium, and large perforations) were created, and different sizes of cartilage plate-TM-coupled complex were built in the simulation. Biomechanical modeling and the optimal thickness of cartilage plate were determined by using FE analysis.

\section{MATERIALS AND METHODS}

A three-dimensional FE model of the human middle ear based on high-resolution computed tomography was recently created by our group. ${ }^{10}$ Experimental results on human or human temporal bone were used to comparatively evaluate our FE model. The experimental data from 64 normal human subjects published by Nishihara and Goode, ${ }^{12} 10$ temporal bones reported by Huber et al., ${ }^{13}$ and stapes footplate displacements obtained from 17 human temporal bones by Gan et al. ${ }^{14}$ were selected for model validation. The results showed that the predicted umbo and stapes displacements across frequencies by our model were very similar to those predicted or measured in these three studies. ${ }^{10} \mathrm{We}$ also developed a cartilage plate-TM-coupled model based on the FE model. ${ }^{11}$ The Raleigh damping parameter, $\beta$, of the cartilage plate at different frequencies and the optimal thickness of cartilage were calculated by using FE analysis. ${ }^{11}$ Geometric models of the perforated TM were generated using Patran software (MSC Software, Perth, Australia). We created perforations in the pars tensa, involving $15 \%, 55 \%$, and $85 \%$ of the area of the TM (Fig. 1). The $85 \%$ perforation approximated $55.2 \mathrm{~mm}^{2}$ of the area of the TM. The remaining TM was composed of three layers of 1,274 eight-noded hexahedral solid elements. The 55\% posterior perforation approximated $35.79 \mathrm{~mm}^{2}$ of the area of the TM. The remaining TM was composed of three layers of 2,549 eight-noded hexahedral solid elements. The $15 \%$ perforation approximated $9.76 \mathrm{~mm}^{2}$ of the area of the TM. The remaining TM was composed of three layers of 4,128 eight-noded hexahedral solid elements. Reconstructed cartilage plates of different sizes
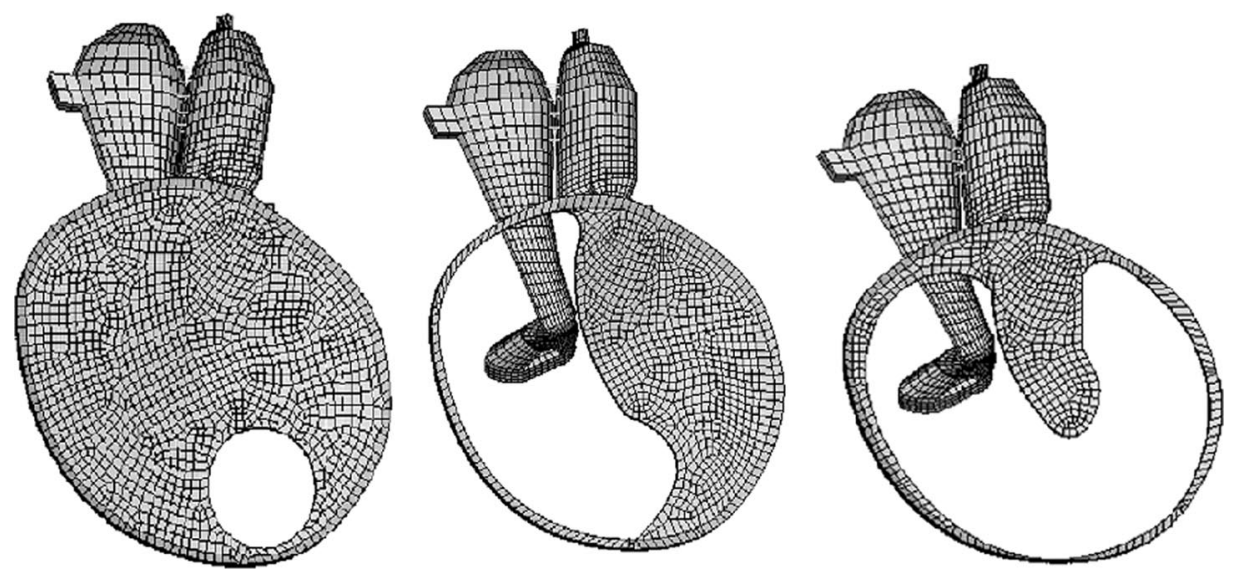

Fig. 1. (Left) Fifteen percent central perforation of tympanic membrane (TM) in pars tensa. Remaining TM was composed of three layers of 4,128 eight-noded hexahedral solid elements. Reconstructed cartilage plate for $15 \%$ central perforation of TM was built by three layers of 570 eight-noded hexahedral solid elements. (Middle) Fifty-five percent posterior perforation of TM in pars tensa. Remaining TM was composed of three layers of 2,549 eight-noded hexahedral solid elements. Reconstructed cartilage plate for $55 \%$ perforation of TM was built by three layers of 2,122 eight-noded hexahedral solid elements. (Right) Eighty-five percent perforation of TM in pars tensa. Remaining TM was composed of three layers of 1,274 eight-noded hexahedral solid elements. Reconstructed cartilage plate for $85 \%$ perforation of TM was built by three layers of 3,426 eight-noded hexahedral solid elements. 
were built using three layers of 3,426 eight-noded hexahedral solid elements for the $85 \%$ perforation, 2,122 eight-noded hexahedral solid elements for the $55 \%$ perforation, and 570 eight-noded hexahedral solid elements for the $15 \%$ perforation. FE analysis involved interpolation betweens nodes. The reasons that we use three layers of cartilage to simulate a graft were as follows. First, there were three layers of TM; for better coupling and interface with the TM, three layers are better than one. Second, three layers of structures would have a greater ability to withstand strain and stress than a single layer. Third, the vibration amplitudes in a mathematical model would be more precise and reasonable than one layer. The cartilage plate was connected to the TM and adjusted to fit the geometry and shape of the TM. The interfaces between the cartilage plate and the TM were directly connected and contained all the necessary degrees of freedom. This means that the interfaces of the computer-generated cartilage plate exactly met the edge of created TM perforation. The cartilage plate was assumed to have isotropic properties and was the same as used in our previous work. ${ }^{14}$ Boundary conditions were also adopted from our previous middle ear study. ${ }^{10-14}$ Poisson's ratio was assumed to be 0.3 for all materials of the system. It has been shown that hyaline articular cartilage of the skeleton has linear viscoelastic material properties. ${ }^{15} \mathrm{We}$ can assume a linear-elastic behavior in the cartilage specimen because of the small strain values and quick recovery during relaxation. Predicted displacement of the stapes footplate and the tip of the malleus induced by sound pressure on the reconstructed TM with 80 $\mathrm{dB}$ sound pressure level were computed from the FE model over the auditory frequency range of 100 to $8,000 \mathrm{~Hz}$ as the force input into the ANSYS software. The vibration amplitudes of different cartilage plate thicknesses were calculated from the simulating FE model.

\section{RESULTS}

Figure 2 shows the predicted vibration amplitude of different thicknesses of cartilage plate (for $85 \%$ perforation of the TM) for the coupled FE model. The vibration amplitude increased with decreasing thickness. Because of the greater mass and stiffness, there was more sound reflection on to the cartilage plates than on to the TM, especially at low frequencies. At lower frequencies $(<2,300$ $\mathrm{Hz}$ ), the $0.2 \mathrm{~mm}$ cartilage plate showed a frequency response function similar to that of a TM in umbo and stapes-footplate displacement (Fig. 2, A and B, respectively). Likewise, at higher frequencies $(>2,300 \mathrm{~Hz})$, the $0.1 \mathrm{~mm}$ cartilage plate showed a frequency response function similar to that of a TM in umbo and stapes-footplate displacement (Fig. 2, C and D, respectively). Figure 3 shows the predicted vibration amplitude of different thicknesses of the cartilage plate (for $55 \%$ perforation of the $\mathrm{TM})$ for the coupled $\mathrm{FE}$ model. At lower frequencies $(<2,300 \mathrm{~Hz})$, the cartilage plate-TM, with a thickness less than $0.2 \mathrm{~mm}$, showed a frequency response function similar to that of a TM in umbo and stapes-footplate displacement (Fig. 3, A and B, respectively). Likewise, at higher
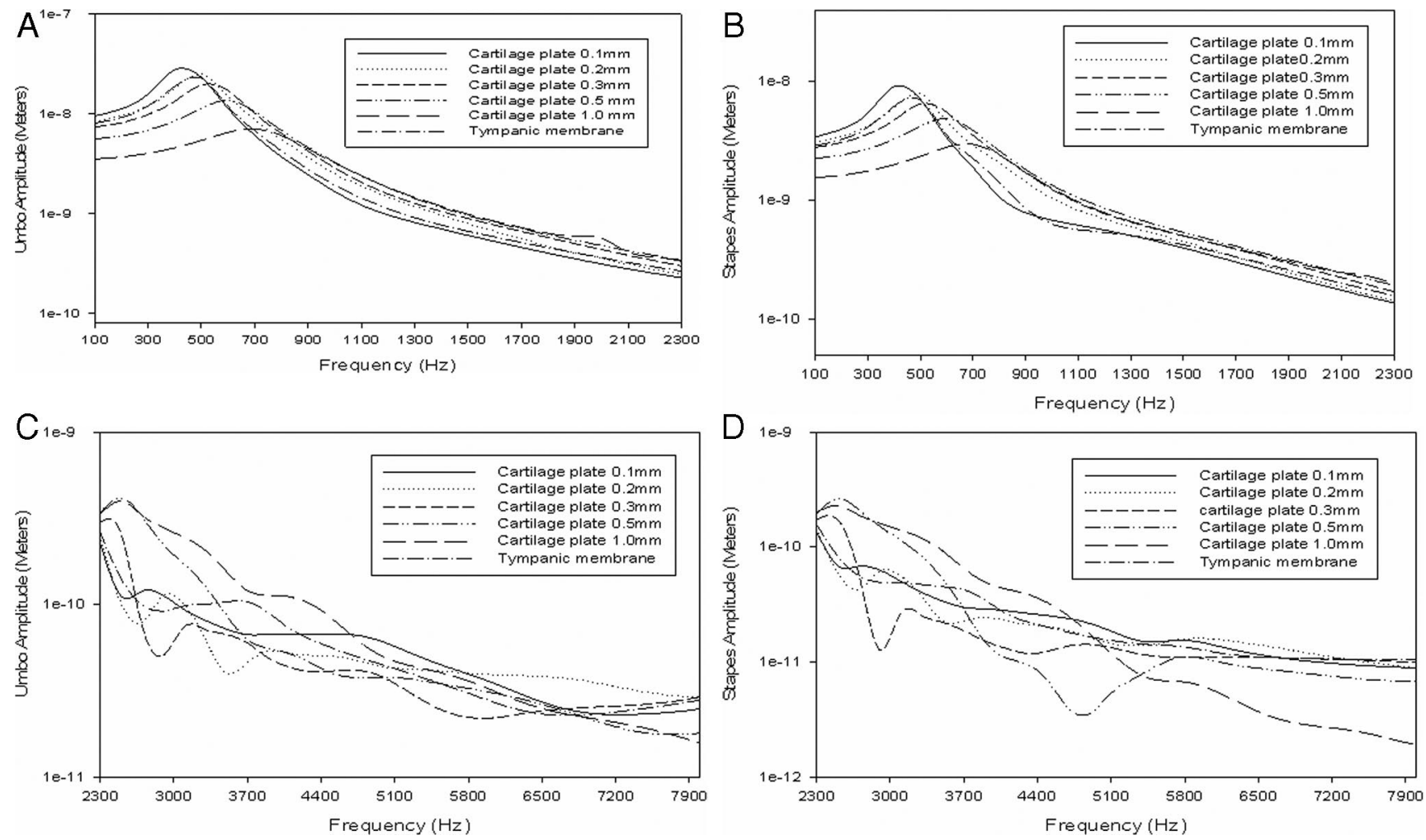

Fig. 2. (A) Predicted frequency response function of umbo versus frequency of acoustic stimuli at $80 \mathrm{~dB}$ sound pressure level (SPL) using cartilage plate-tympanic membrane (TM)-coupled finite element model with varying thickness in frequency range of 100 to $2,300 \mathrm{~Hz}(85 \%$ cartilage plate-TM complex). (B) Predicted frequency response function of stapes footplate versus frequency of acoustic stimuli at $80 \mathrm{~dB}$ SPL using cartilage plate-TM-coupled finite element model with varying thickness in frequency range of 100 to $2,300 \mathrm{~Hz}(85 \%$ cartilage plate-TM complex). (C) Predicted frequency response function of umbo versus frequency of acoustic stimuli at $80 \mathrm{~dB}$ SPL using cartilage plate-TMcoupled finite element model with varying thickness in frequency range of 2,300 to $8,000 \mathrm{~Hz}(85 \%$ cartilage plate-TM complex). (D) Predicted frequency response function of stapes footplate versus frequency of acoustic stimuli at $80 \mathrm{~dB}$ SPL using cartilage plate-TM-coupled finite element model with varying thickness in frequency range of 2,300 to $8,000 \mathrm{~Hz}$ ( $85 \%$ cartilage plate-TM complex). 

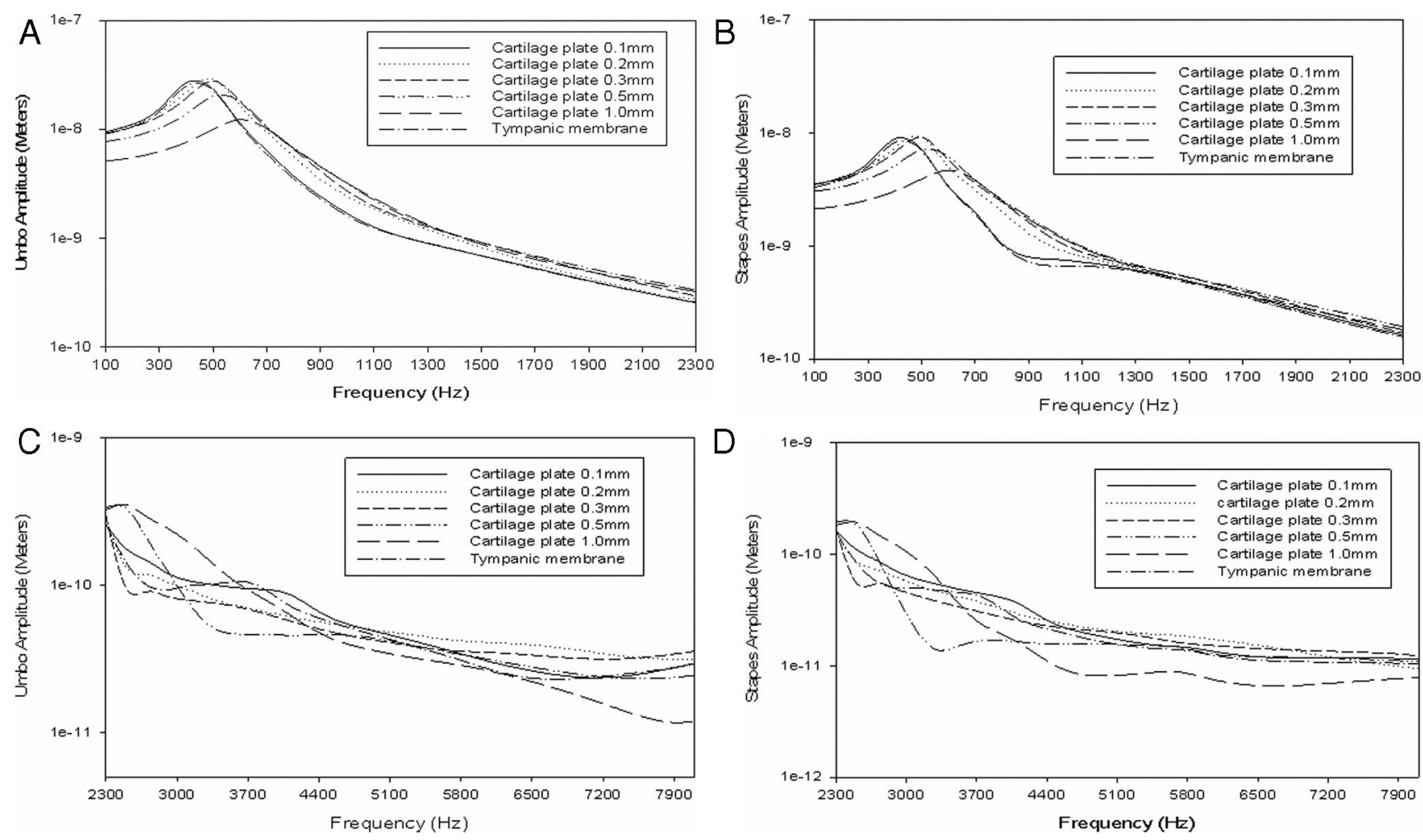

Fig. 3. (A) Predicted frequency response function of umbo versus frequency of acoustic stimuli at $80 \mathrm{~dB}$ sound pressure level (SPL) using cartilage plate-tympanic membrane (TM)-coupled finite element model with varying thickness in frequency range of $100 \mathrm{to} 2,300 \mathrm{~Hz}(55 \%$ cartilage plate-TM complex). (B) Predicted frequency response function of stapes footplate versus frequency of acoustic stimuli at $80 \mathrm{~dB}$ SPL using cartilage plate-TM-coupled finite element model with varying thickness in frequency range of 100 to $2,300 \mathrm{~Hz}(55 \% \mathrm{cartilage}$ plate-TM complex). (C) Predicted frequency response function of umbo versus frequency of acoustic stimuli at $80 \mathrm{~dB}$ SPL using cartilage plate-TMcoupled finite element model with varying thickness in frequency range of 2,300 to $8,000 \mathrm{~Hz}$ (55\% cartilage plate-TM complex). (D) Predicted frequency response function of stapes footplate versus frequency of acoustic stimuli at $80 \mathrm{~dB}$ SPL using cartilage plate-TM-coupled finite element model with varying thickness in frequency range of 2,300 to $8,000 \mathrm{~Hz}$. (55\% cartilage plate-TM complex).

frequencies $(>2,300 \mathrm{~Hz})$, the cartilage plate-TM with a thickness of less than $0.2 \mathrm{~mm}$ showed a frequency response function similar to that of a TM in umbo and stapes-footplate displacement (Fig. 3, C and D, respectively). Figure 4 shows the predicted vibration amplitude of different thicknesses of the cartilage plate (for $15 \%$ perforation of the TM) for the coupled FE model. The vibration amplitude also increased with decreasing thickness. At lower frequencies $(<2,300 \mathrm{~Hz})$, the cartilage plate-TM model with a thickness of less than $1.0 \mathrm{~mm}$ showed a frequency response function similar to that of a TM in umbo and stapes-footplate displacement (Fig. 4, A and $\mathrm{B}$, respectively). Likewise, at higher frequencies $(>2,300 \mathrm{~Hz})$, the cartilage plate-TM model with a thickness of less than $1.0 \mathrm{~mm}$ showed a frequency response function similar to that of a TM in umbo and stapesfootplate displacement (Fig. 4, C and D, respectively).

\section{DISCUSSION}

The use of cartilage for reconstruction of the TM has been established particularly for cases of chronic tubal dysfunction, adhesive processes, and total or recurrent defects of the TM. ${ }^{16-18}$ In these cases, cartilage can offer a better prognosis for permanent closure in comparison with temporalis fascia and perichondrium, which are used pre- dominantly for TM reconstruction in the normal, ventilated middle ear. Because the increased mass and stiffness of a cartilage-reconstructed eardrum might adversely affect its sound-wave transfer characteristics, its acoustic properties should be considered in making a choice of material. Clinical studies may be misleading because of confounding variables such as middle ear inflammation, persistent tubal dysfunction, and different surgical techniques used. Various factors are likely to influence the sound transmission characteristics of the cartilage plate. Material properties such as mass, density, and Young's modulus, as well as the thickness of the grafted tissue, will determine its acoustic quality, with the latter two defining the stiffness of the cartilage plate. In addition, surgical processing of the cartilage might further affect the acoustic properties of the transplant because the shape and position of the transplant within the TM are decisive factors. To exclude these factors, we developed a cartilage plate-TM-coupled model with different sizes of TM perforation to determine the basic mechanical and acoustic behaviors of reconstructed cartilage plate compared with the TM in this study. This model offers the advantages of reproducible test conditions and diminished additional confounding parameters and keeps the boundary conditions constant. 

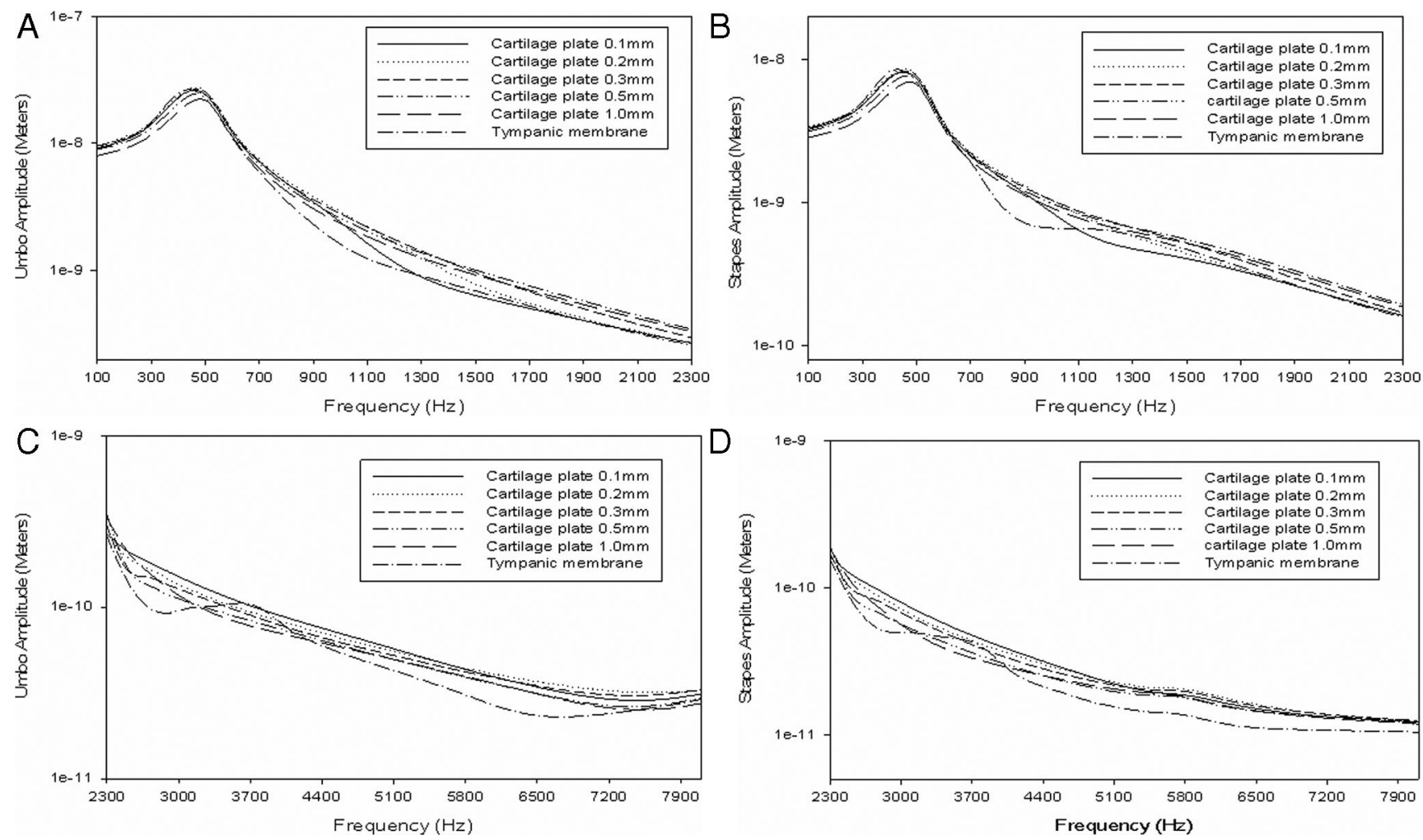

Fig. 4. (A) Predicted frequency response function of umbo versus frequency of acoustic stimuli at $80 \mathrm{~dB}$ sound pressure level (SPL) using cartilage plate-tympanic membrane (TM)-coupled finite element model with varying thickness in frequency range of 100 to $2,300 \mathrm{~Hz}(15 \%$ cartilage plate-TM complex). (B) Predicted frequency response function of stapes footplate versus frequency of acoustic stimuli at $80 \mathrm{~dB}$ SPL using cartilage plate-TM-coupled finite element model with varying thickness in frequency range of 100 to $2,300 \mathrm{~Hz}(15 \% \mathrm{cartilage}$ plate-TM complex). (C) Predicted frequency response function of umbo versus frequency of acoustic stimuli at $80 \mathrm{~dB}$ SPL using cartilage plate-TMcoupled finite element model with varying thickness in frequency range of 2,300 to $8,000 \mathrm{~Hz}(15 \%$ cartilage plate-TM complex). (D) Predicted frequency response function of stapes footplate versus frequency of acoustic stimuli at $80 \mathrm{~dB}$ SPL using cartilage plate-TM-coupled finite element model with varying thickness in frequency range of 2,300 to $8,000 \mathrm{~Hz}$ (15\% cartilage plate-TM complex).

Cartilage may adversely affect the transfer characteristics of the TM by its increased mass and, especially, its stiffness. The optimal thicknesses of cartilage plates were determined by different sizes of transplants and different frequencies. In our model, the cartilage plate was directly connected to the TM. With decreased thickness, the transfer qualities improved. ${ }^{19}$ Small cartilage transplants, embedded in a normally vibrating TM, will influence the vibration pattern of the TM less than large cartilage transplants. Larger cartilage plates might influence the transfer behavior in the entire frequency range because the bending stiffness of the entire TM increases with the size of the implanted cartilage pieces. ${ }^{7}$ If a larger cartilage plate is used for reconstruction, a smaller thickness of transplant is necessary. For optimal acoustic transfer behavior, the cartilage should be cut as thinly as possible. Overbosch ${ }^{20}$ was the first to describe a microslice technique to improve the acoustic properties of the reconstructed TM. Using a dermatome, he cut the cartilage into a plate with a thickness of 0.1 to $0.2 \mathrm{~mm}$. Currently, thin cartilage plates are used for reconstructing total TM defects and for protecting total or partial ossicular replacement prosthesis implants underneath the TM. Cartilage is well tolerated by the TM, can be placed under some degree of tension beneath the graft, and has a very low risk of extrusion. Altenau and Sheehy ${ }^{21}$ reported no cases of cartilage resorption and only one instance of extrusion in 564 procedures.

A study using the laser Doppler interferometer and cadaver cartilage conducted by Mürbe et al.7,19 demonstrated that the ideal acoustic thickness of cartilage should be approximately $0.5 \mathrm{~mm}$. A major limitation of a temporal bone model is that it is an unloaded model, without connection of the cartilage transplants to the ossicular chain and the inner ear. Mürbe et al.'s results only allow comparison between the different investigated cartilage transplants, which might differ in certain parameters from the human ear.

On the basis of our analysis, different thicknesses of cartilage transplants should be used in cartilage myringoplasty for different sizes of TM perforation. For medium and large TM perforations, the optimal thickness of cartilage graft in myringoplasty appears to be 0.1 to $0.2 \mathrm{~mm}$. For small perforations, cartilage less than $1.0 \mathrm{~mm}$ are a good compromise between mechanical stability and low acoustic transfer loss. A major challenge involves the prediction of the eustachian tube function preoperatively. This has been the subject of numerous investigations, the general consensus being that no specific examination reliably predicts tubal function. ${ }^{22}$ Dornhoffer ${ }^{23}$ compared 
perichondrium and cartilage in revision type I tympanoplasty and showed no difference in hearing between the two groups, with both exhibiting an air-bone gap of less than $10 \mathrm{~dB}$. Although he utilized full-thickness grafts. He did not indicate the exact thickness of the cartilage. Another comparison study was performed by Gerber et al., ${ }^{24}$ in which cartilage was compared with fascia in a frequencyspecific manner, and no significant difference was seen. The exact thickness of cartilage plate was also not described in detail. Furthermore, the hearing gains in air conduction of cartilage group were smaller than those of fascia group.

In summary, to control some variables, we created a cartilage plate-TM-coupled model with different sizes of TM perforation. This model only offers the optimal graft thickness of cartilage plate for myringoplasty under theoretic and ideal conditions. The real results could be affected by confounding variables including middle ear inflammation, eustachian tube function, and the mobility status of the ossicular chains. Therefore, clinical studies with a larger population and long-term follow-up are needed. Our model may be improved in several respects, such as by simulating middle ear alterations caused by middle ear pressure changes. It is expected that future studies will determine how alterations in the structure and mechanical properties in normal and pathologic conditions of the human ear can affect acoustic transmission through the external ear canal and middle ear to inner ear.

\section{BIBLIOGRAPHY}

1. Nissen AJ, Nessen RL, Younkers AJ. A historical review of the use of bone and cartilage in otologic surgery. Ear Nose Throat J 1986;65:493-496.

2. Storrs LA. Myringoplasty with the use of fascia grafts. Arch Otolaryngol 1961;74:45-49.

3. Shea JJ. Vein graft closure of eardrum perforation. Arch Otolaryngol 1960;72:445-447.

4. Preobrazhenski TB, Rugov AA. The employment of preserved dura graft in tympanoplasty. Vestn Otorhinolaringol 1965; 5:38-42.

5. Sheehy JL, Glasscock ME. Tympanic membrane grafting with temporalis fascia. Arch Otolaryngol 1967;86:391-402.

6. Jansen C. Cartilage-tympanoplasty. Laryngoscope 1963;73: 1288-1302.

7. Mürbe D, Zahnert T, Bornitz M, et al. Acoustic properties of different cartilage reconstruction techniques of the tympanic membrane. Laryngoscope 2002;112:1769-1776.
8. Beer HJ, Bornitz M, Harstke HJ, et al. Modeling of components of the human middle ear and stimulation of their dynamic behavior. Audiol Neurotol 1999;4:156-162.

9. Funnell WR, Khanna SM, Decramemer WF. On the degree of rigidity of the manubrium in a finite element model of the cat ear drum. J Acoust Soc Am 1992;91:2082-2090.

10. Lee CF, Chen PR, Lee WJ, et al. Three-dimensional reconstruction and modeling of middle ear biomechanics by high-resolution computed tomography and finite element analysis. Laryngoscope 2006;116:711-716.

11. Lee CF, Hsu LP, Chen PR, et al. Biomechanical modeling and design optimization of cartilage myringoplasty using finite element analysis. Audiol Neurotol 2006;11:380-388.

12. Nishihara S, Goode RL. Measurement of tympanic membrane vibration in 99 human ears. In: Hüttenbrink KB, ed. Middle Ear Mechanics in Research and Otosurgery. Dresden, Germany: Dresden University of Technology, 1996:91-93.

13. Huber A, Ball G, Asai M, Goode R. The vibration pattern of the tympanic membrane after placement of a total ossicular replacement prosthesis. In: Hüttenbrink KB, ed. Proceedings of the International Workshop On Middle Ear Mechanics in Research and Otosurgery, Dresden, Germany: Dresden University of Technology, 1997:219-222.

14. Gan RZ, Sun Q, Robert KD Jr, et al. Three-dimensional modeling of middle ear biomechanics and its applications. Otol Neurotol 2002;23:271-280.

15. Woo SL, Akeson WH, Jemmot GF. Measurement of nonhomogenous, directional mechanical properties of articular cartilage in tension. J Biomech 1976;9:785-791.

16. Dornhoffer JL. Surgical management of the atelectatic ear. Am J Otol 2000;21:315-321.

17. Heermann HJ, Heermann H, Kopstein E. Fascia and cartilage palisade tympanoplasty: nine years experience. Arch Otolaryngol 1970;91:229-240.

18. Hildman H, Luckhaupt H, Schmelzer A. Die Verwendung von Knorpel in der Mittelohrchirurige. HNO 1996;44:597-603.

19. Zahnert T, Hüttenbrink KB, Mürbe D, et al. Experimental investigation of the use of cartilage in tympanic membrane reconstruction. Am J Otol 2000;21:322-328.

20. Overbosch HC. Homograft myringoplasty with microsliced septal cartilage. Proc Otorhinolaryngol 1971;33:356-357.

21. Altenau MM, Sheehy JL. Tympanoplasty: cartilage prosthesis: a report of 564 cases. Laryngoscope 1978;88:895-904.

22. Cohen AM, Schwaber MK, Anthony LS, et al. Eustachian tube function and tympanoplasty. Ann Otol Rhinol Laryngol 1979;88:339-347.

23. Dornhoffer JL. Hearing results with cartilage tympanoplasty. Laryngoscope 1997;107:1094-1099.

24. Gerber MJ, Mason JC, Lambert PR. Hearing results after primary cartilage tympanoplasty. Laryngoscope 2000;110: 1994-1999. 